\title{
SBgr
}

\section{Space Weather and the Global Muon Detector Network - GMDN}

Nelson Jorge Schuch [1], Kazuoki Munakata [2], John W. Bieber [3], Marc. L. Duldig [4], Ismail Sabbah [5], Alisson Dal Lago [6], Adriano Petry [1], Nivaor Rodolfo Rigozo [1], Marlos Rockenbach da Silva [7],Carlos Roberto Braga [6],

Lucas Ramos Vieira [6], Tardelli Ronan Coelho Stekel [6], Mauricio Rosa Souza [1], Bruno Knevitz Hammerschmitt [1], Roger Hatwig de Lima [1].

([1] Southern Regional Space Research Center - CRS/CCR/INPE - MCT in collaboration with the Space Science Laboratory of Santa Maria - LACESM/CT - UFSM, Santa Maria, RS, Brazil, njschuch@lacesm.ufsm.br; [2] Shinshu University,

Matsumoto, Japan; [3] University of Delaware, Delaware, United States; [4] School of Mathematics and Physics, University of Tasmania, Tasmania, Australia; [5] Department of Natural Sciences, Collage of Health Sciences, Public Authority of Applied Education and Training, Kuwait; [6] National Institute for Space Research - DGE/CEA/INPE, São José dos Campos, SP, Brazil; [7] Universidade Vale do Paraiba, São José dos Campos, Brazil;).

Copyright 2011, SBGf - Sociedade Brasileira de Geofísica

This paper was prepared for presentation during the $12^{\text {th }}$ International Congress of the Brazilian Geophysical Society held in Rio de Janeiro, Brazil, August 15-18, 2011.

Contents of this paper were reviewed by the Technical Committee of the $12^{\text {th }}$ International Congress of the Brazilian Geophysical Society and do not necessarily represent any positon or the SBGi, its orlicers or members. Elecronic reproduction or

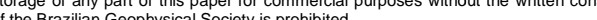

\section{Abstract}

The main objective of this work is to present an overview of the space weather and its relation with the global network of ground-based multi-directional muon detectors (GMDN). The GMDN is an international collaboration consisting of 10 institutions from 6 countries in 5 continents. A multidirectional muon detector for measuring high-energy galactic cosmic rays (GCRs) was installed in 2001 and expanded in its detection area in 2005, through an international cooperation between Brazil, Japan and USA, and has been in operation since then at the Southern Space Observatory - SSO/CRS/INPE -MCT, $\left(29,4^{\circ} \mathrm{S}, 53,8^{\circ} \mathrm{W}, 480 \mathrm{~m}\right.$ a.s.I), São Martinho da Serra, RS, in southern Brazil, as an important component of the GMDN. The observations conducted by this detector are used for forecasting the arrival of the interplanetary coronal mass ejections (ICMEs) and the geomagnetic storms at the Earth. The detector measures high-energy GCRs by detecting secondary muons produced from the hadronic interactions of primary GCRs (mostly protons) with atmospheric nuclei. While muons have a relatively short life-time (about 2.2 microseconds at rest), they can reach the detector at ground level because of the relativistic effect of the time dilation with heir high speed ( $0.96 \mathrm{c})$, preserving the incident direction of primary particles. The multi-directional detector can measure the GCR intensity in various directions at a single location, such as SSO in Brazil. ICMEs accompanied by a strong shock often forms a GCR depleted region behind the shock. The Forbush decrease is observed when the Earth enters in this depleted region. Some particles from this depleted region leak into the downstream, traveling with almost the speed of light, much faster than the approaching shock, and creating the precursory loss-cone anisotropy around the sunward IMF direction at the Earth. Loss-cones are typically visible 4-8 hours prior to the shock arrival and the onset of major geomagnetic storm at the Earth. This cosmic-ray precursor can be detected sometimes as early as ten hours prior to the shock arrival at the Earth. With the real time data from the upgraded GMDN, its methodology and data reduction techniques permits accurate Space Weather forecasting.

\section{Introduction}

The main objective of this work is to present an overview of the ground-based Global multi-directional Muon Detectors Network (GMDN). A Multi-directional Muon Detector (MMD) for measuring high-energy, $\sim 50 \mathrm{GeV}$, galactic cosmic rays (GCRs) was installed in 2001 and expanded in its detection area in 2005, through an international cooperation between Brazil, Japan and USA and has been in operation since then at the Southern Space Observatory - SSO/CRS/CCR/INPE - MCT, $\left(29,4^{\circ}\right.$ S, 53, $8^{\circ}$ W, 480 m a.s.I), São Martinho da Serra, $\mathrm{RS}$, in southern Brazil, as an important component of the global network of ground-based multi-directional muon detectors - GMDN. The observations conducted by these detectors and network are used for forecasting the arrival of the Interplanetary Coronal Mass Ejections (ICMEs) and the geomagnetic storms at the Earth.

\section{The physical mechanism}

ICMEs accompanied by a strong shock often forms a GCR depleted region behind the shock. The Forbush decrease is observed when the Earth enters in this depleted region. Some particles from this depleted region leak into the downstream, traveling with almost the speed of light, much faster than the approaching shock, and creating the precursory loss-cone anisotropy around the sunward Interplanetary Magnetic Field (IMF) direction at the Earth, Fig 1. 
Space Weather and the Global Muon Detector Network - GMDN

\section{Methodology}

The MMDs detectors measure high-energy GCRs

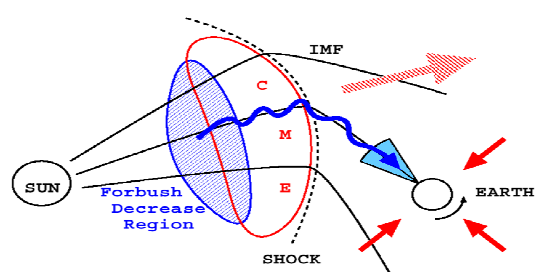

Fig. 1: Physical mechanism causing loss-cone precursors $^{1,2}$.

( $50-170 \mathrm{GeV})$ by detecting secondary muons produced from the hadronic interactions of primary GCRs (mostly protons and heavier nuclei) with atmospheric nuclei. While muons have a relatively short life-time (about 2.2 microseconds at rest), they can reach the MMD at ground level because of the relativistic effect of the time dilation with their high speed ( 0.96c), preserving the incident direction of primary particles, Fig. 2 and Fig. 3.

\section{Observations}

Since the large detector mass is required for detecting high energy cosmic rays, ground-based instruments suh as MMDs remain the best and cheapest method for observing and studying these particles ${ }^{1,2}$. MMDs can record secondary cosmic rays created by the interactions of $>1 \mathrm{GeV}$ primary cosmic rays with Earth's atmosphere ${ }^{3}$

The multi-directional Muon detectors, MMDs, can measure the GCR intensity in various directions at a single location, such as at SSO in southern Brazil, Figure 4.

The sky coverage of the Global Muon Detector Network - GMDN

Currently, the Global multi-directional Muon Detectors Network - GMDN is consisted by four MMDs: at Nagoya (Japan), Hobart (Australia), São Martinho da Serra, RS (Brazil) and Kuwait City (Kuwait). The GMDN can continuously monitor the GCR intensity in 60 directional channels covering almost the entire sky and can precisely measure the variation of the GCR streaming separately from the variation of the GCR density, i.e. the isotropic component of the GCR intensity.

The geographic location (big star) of each detector is presented in Fig. 5. Each of symbols (squares, triangles and circles) shows the existing asymptotic viewing direction of a particle incident to each MMD with the median primary rigidity, after correction for geomagnetic bending of cosmic ray orbits.

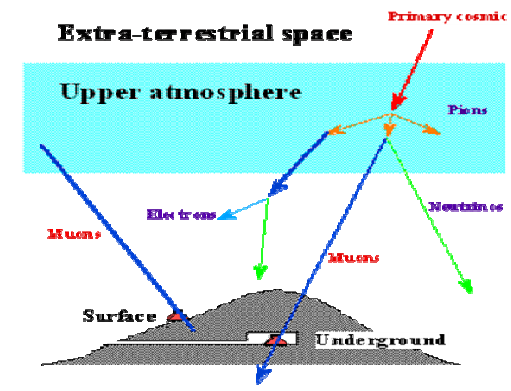

Fig. 2: Ground-based detectors measure byproducts of the interaction of primary cosmic rays with Earth's atmosphere.

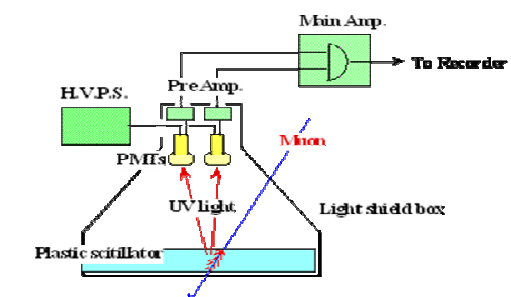

Fig. 3: The SSO's Muon detector'.

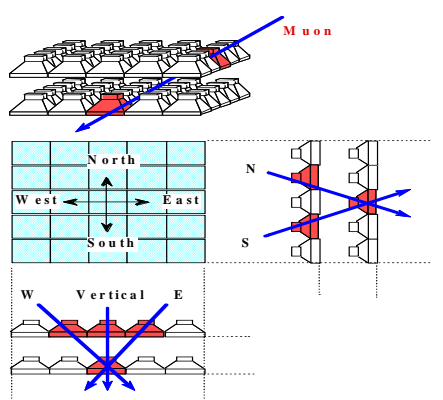

Fig. 4: The multi-directional Muon detector, MMD, can measure the GCR intensity in various directions at a single location ${ }^{4}$.

The number of directional channels monitored by each MMD is: Nagoya - 17; Hobart - 13; Kuwait - 13 and São Martinho da Serra - 17 (V, N, S, E, W, NE, NW, SE, SW, N2, S2, E2, W2, N3, S3, E3, W3). The track through each symbol represents the spread of viewing directions 
cosmic ray intensity relative to the average, and the diameter of each circle is proportional to the magnitude of deficit or excess ${ }^{5}$

With the GMDN the loss-cones are typically visible 4-8 hours prior to the shock arrival and the onset of major geomagnetic storm at the Earth ${ }^{5}$.

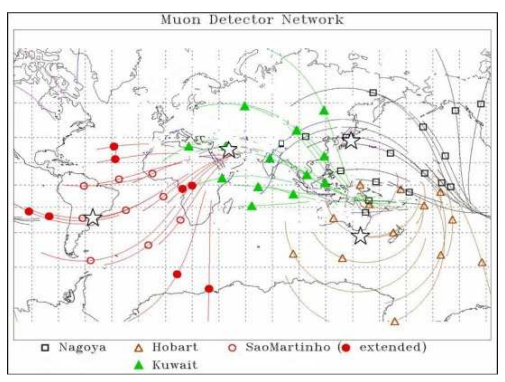

Fig. 5: The GMDN's MMD geographic location (big star) $)^{5}$.

corresponding to the central $80 \%$ of each multi-directional detector's energy response.

Therefore, with a suitable analysis, the ground-based MMDs observations combined with its global network GMDN data, can provide an unique information on the conditions in the near-Earth interplanetary medium, solarterrestrial relations and space weather ${ }^{3}$.

\section{Results}

Munakata et $a l^{5}$ examined cosmic ray precursors of geomagnetic storms by analyzing the pitch angle distribution of GCR intensity measured by a couple of MMDs at Nagoya and Hobart. Out of 22 storms with good coverage, they found $15(68,2 \%)$ storms associated with precursors, of which 10 were the loss-cone precursors.

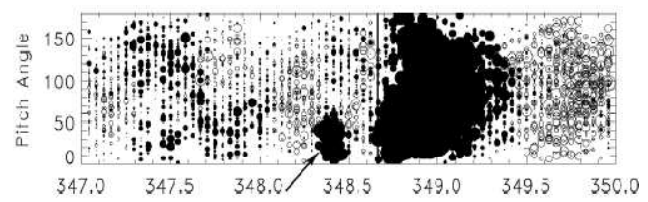

Fig. 6: Visualization of precursors using the methodology developed by $\mathrm{Da}$ Silva ${ }^{6}$ for a geomagnetic storm on December $14,2006^{6}$.

Each circle on Figure 6 represents an hourly count rate in a single directional channel plotted at the appropriate time (abscissa) and pitch angle (ordinate) of the viewing direction. A pitch angle of $0^{\circ}$ corresponds to the sunward direction along the IMF. Open and solid circles represent, respectively, an excess and deficit of
This cosmic-ray precursor can be detected sometimes as early as ten hours prior to the shock arrival at the Earth ${ }^{5}$.

Despite been observed more frequently before intense geomagnetic storms (see Munakata et $\mathrm{al}^{5}$ ), loss-cones can also be observed in small or moderate geomagnetic storms. One example of a loss-cone observed during a moderate storm in November 15th 2008 is shown in Figure 7. Red circles mean decreases and blue circles mean increases. The diameter of each circle is proportional to the magnitude of deficit or excess. In this case the precursor is observed between 10 and 5 hours before the storm sudden commencement (SSC). In a set of 16 moderate and small geomagnetic storms observed in 2008 by the GMND, Braga ${ }^{7}$ observed precursor signatures before the SSC in 14 (87\%) of the events.

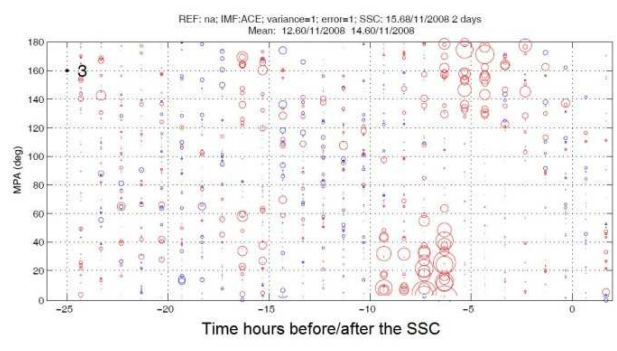

Fig. 7: Visualization of precursors using the methodology developed by $\mathrm{Braga}^{7}$ for a geomagnetic storm on November 15,2008 . In the x-axis the time before/after the SSC is indicated and in the $y$-axis the pitch angle (degrees) is indicated ${ }^{7}$.

\section{The SSO's MMD Observations}

An example of a Geomagnetic storm observed by the SSO's MMD in October 2003 is shown in Fig. 8.

A loss-cone signature observed by the SSO' MMD prior to the SSC causing a severe geomagnetic storm is shown in Fig. 9. The loss cone has all the right characteristics, $3 \mathrm{hr}$ duration, implying $\sim 45 \mathrm{deg}$ width, and it onsets first in the eastward viewing channel, then vertical and westward,_Fushishita et $\mathrm{al}^{10}$.

Real time plots of the pitch angle distribution, the bidirectional streaming and the GCR flow direction deduced from the GMDN are available in the GMDN Space Weather Prediction with Cosmic Rays Website (http://neutronm.bartol.udel.edu/spaceweather/).

The Brazilian National Institute for Space Research INPE/MCT developed a program to monitor the Space 


\section{Space Weather and the Global Muon Detector Network - GMDN}

Weather Solar Terrestrial interactions by satellite and ground-based observations, and to provide useful information to space related communities, technological, industrial and academic areas. It is aimed to observe physical parameters of the geoenvironment, $\mathrm{CME}$

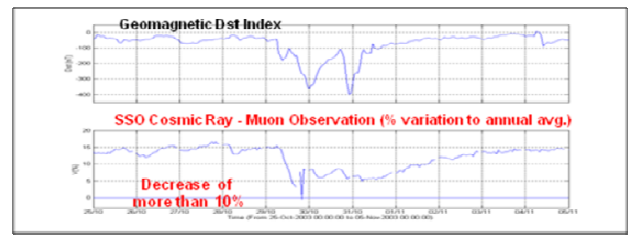

Fig. 8: Geomagnetic storm observed by the SSO's MMD in October 2003.

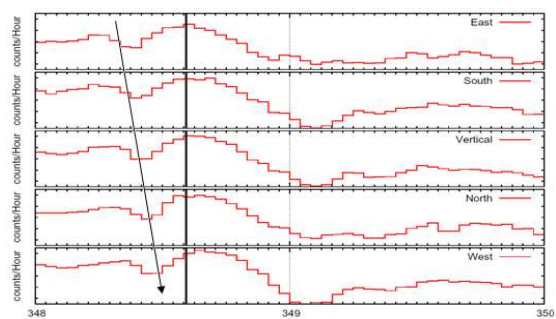

Fig. 9: Forbush decrease observed by the SSO's MMD in December 14, 2006.

information by satellite and ground-based cosmic ray monitoring at the SSO's MMD. The INPE's Space Weather Website (http://www.inpe.br/spaceweather/) is available for real time monitoring.

The real time processing of GMDN data with $5 \mathrm{CR}$ multi-directional muon detectors, MMDs, are been processed at the Institute of Space Systems from DLR ${ }^{8}$ Bremen, Germany, an example is presented in Fig. 10.

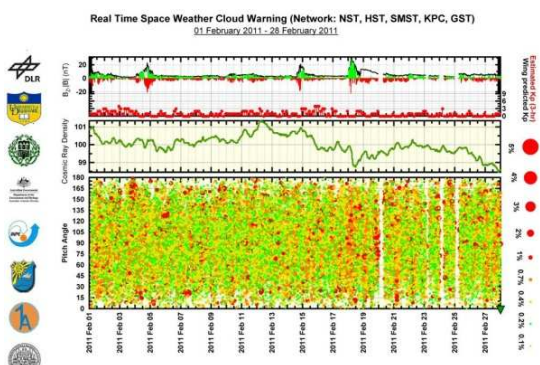

Fig. 10: Real time processing of GMDN data with $5 \mathrm{CR}$ multi-directional muon detectors ${ }^{8}$.

It is also important to mention the Solar TErrestrial RElations Observatory - STEREO Space Weather Page (http://secchi.nrl.navy.mil/spwx/) where are available real time monitoring from the Sun and interplanetary medium.

\section{Conclusions}

The MMDs observations and results from Kuwabara

et $\mathrm{al}^{9}$ and Fushishita et $\mathrm{al}^{10}$ show that the GMDN is a high-quality tool for Space Weather monitoring and forecasting.

With the expectation of the approval by European Commission of the NExt generation Space TEChnology NESTEC Project, the GMDN may be upgraded in 2010/2011 including new muon detectors in Bremen, Germany and in Hermanus, South Africa, and it will become an international collaboration consisting of 10 institutions from 6 countries in 5 continents.

With the real time data from the upgraded GMDN, the methodology and the technique for applied international services will permit very accurate Space Weather forecasting in near future.

For future plans it is suggested the combination of real time data and other information from NASA's STEREO and from the Solar Dynamics Observatory - SDO missions with that from GMDN, in order to develop a new methodology and technique for applied international services, to permit Solar Heliosphere 3-D visualization with accurate Space Weather forecasting. Possible support for the future plans may come from: USA (NSF), BRAZIL (FAPESP, MCT (FINEP - CNPq)), EU (ESA) and JAPAN (JAXA).

\section{Acknowledgements}

The authors acknowledge the USA's National Science Foundation (NFS) for a subcontract between the University of Delaware and FATEC/UFSM from Brazil, for supporting part of the upgrade of the SSO's MMD in 2005. Thanks to the Federal University of Santa Maria and its Center of Technology (LACESM/CT-UFSM) for supporting the GMDN. The GMDN is also supported by the Shinshu University and the Nagoya University in Japan, the University of Tasmania and the Australian Antarctic Division, in Australia, the University of Delaware and the NSF, in USA, the Kuwait University, Kuwait, the Alexandria University, Egypt, and Dr. Frank Jansen from the DLR Institute of Space Systems, Germany.

\section{References}

${ }^{1}$ Simpson, J. A., W. Fonger and S. B. Treiman, Cosmic radiation intensity-time variations and their origin. I. Neutron intensity. Variation method and meteorological factors, Phys. Rev., 90, 934-950, 1953.

2 McDonald, F. B., Integration of neutron monitor data with spacecraft observations: A historical perspective, Space Sci. Rev., 93, 263-284, 2000. 
${ }^{3}$ Kuwabara, T., Bieber, J. W., Clem, J., Everson, P., Ryle, R., Munakata, K., Yasue, S., Kato, C., Akahane, S. Koyama, M., Fujii, Z., Duldig, M. L, Humble, J. E., Da Silva, M. R., Trivedi, N. B., Gonzalez, W. D., Schuch, N. J. Real-time cosmic ray monitoring system for space weather, Space Weather, 4, S08001, 2006.

${ }^{4}$ Munakata, K.| Shinshu University, Matsumoto, Japan Private Communication.

5 Munakata K., Bieber, J. W., Yasue, S., Kato, C., Koyama, M., Akahane, S., Fujimoto, K., Fujii, Z., Humble, J. E., Duldign M., L.. J. Geopghys. Res.105, 7457, 2000.

${ }^{6}$ Da Silva, M. R. Ph.D. Thesis, National Institute for Space Research, São José dos Campos, SP, Brazil, 2010.

${ }^{7}$ Braga, C. R. Mc.S. Thesis, National Institute for Space Research, São José dos Campos, SP, Brazil, 2011.

${ }^{8}$ Frank Jansen, Jörg Behrens, Stanislav Pospisil, Karel Kudela, Space situational awareness satellites and ground based radiation counting and imaging detecto technology, Nuclear Instruments and Methods in Physics Research A, 2011, in press. Journal homepage: www.elsevier.com/locate/nima.

${ }^{9}$ Kuwabara, T., Bieber, J. W., Evenson, P., Munakata, K., Yasue, S., Kato, C., Fushishita, A., Tokumaru, M., Duldig, M. L., Humble, J. E., Silva, M. R., Dal Lago, A., and Schuch, N. J.. Determination of interplanetary coronal mass ejection geometry and orientation from groundbased observations of galactic cosmic rays. Journal of Geophysical Research, v. 114, p. A05109, 2009.

${ }^{10}$ Fushishita, A., Kuwabara, T., Kato, C., Yasue, S., W. Bieber, J., Evenson, P., Da Silva, M. R., Dal Lago, A., Schuch, N. J., Tokumaru, M., Duldig, M. L., Humble, J. E., Sabbah, I., Al Jassar, H. K., Sharma, M. M., and Munakata, K.. Precursors of the Forbush decrease on 2006 December 14 observed with the Global Muon Detector Network (GMDN). The Astrophysical Journal, 715:1239-1247, 2010 